\title{
A POLÍTICA NACIONAL DE ATENÇÃO BÁSICA NO PIAUÍ: uma análise com foco na equidade
}

\author{
Jorge Otavio Maia Barreto \\ Fundação Oswaldo Cruz (FIOCRUZ) \\ Inez Sampaio Nery \\ Universidade Federal do Piauí (UFPI)
}

A POLIITICA NACIONAL DE ATENÇÃO BÁSICA NO PIAUÍ: uma análise com foco na equidade

Resumo: $O$ estudo analisa a implementação da Política Nacional de Atenção Básica no Estado do Piauí com o objetivo de discutir a equidade do acesso à saúde no Piauí, nos anos de 2006 a 2011, a partir da alocação dos recursos federais em saúde e o acesso a ações de Atenção Básica, em especial as consultas médicas para a população geral, infantil e de gestantes. Utiliza estatística descritiva para analisar os indicadores municipais agregados em quintis de renda média domiciliar per capita municipal. Analisa a evolução das transferências federais do Bloco da Atenção Básica do Piauí e utiliza um modelo de regressão linear múltipla para estimar se parâmetros socioeconômicos orientaram a alocação dos recursos federais. Verifica melhoria dos indicadores de acesso avaliados, mas a principal mudança observada foi 0 aumento da disponibilidade de recursos federais para os municípios, especialmente no componente variável do financiamento da Atenção Básica. Não foi possível identificar associação entre as transferências de recursos federais da saúde e critérios de alocação equitativa, com base em indicadores socioeconômicos.

Palavras-chave: Política de saúde, SUS, Atenção básica, Financiamento em saúde, Equidade.

NATIONAL POLICY OF PRIMARY HEALTH CARE IN PIAUÍ: an analysis focused on equity

Abstract: The implementation of the National Policy of Primary Care in Piaui State was analyzed with the objective to discuss equity to access health, in 2006 to 2011, taking the allocation of federal resources and access to Primary Health Care, especially medical appointments for the general population, children and pregnant women. Was used descriptive statistics to analyze municipal indicators aggregated into quintiles of household income. The study analyzed the evolution of federal transfers for the Primary Health Care in Piauí and used a linear regression model to estimate the effect of socioeconomic parameters on the allocation of federal resources. It has been found improving in the evaluated access indicators but the main change was the increased availability of federal resources for the municipalities, especially in the variable component financing of Primary Health Care. It was not possible to identify association between the federal transfers and the analyzed socioeconomic criteria for an equitable allocation based on the socioeconomic indicators.

Key words: Health policy, SUS, Primary health care, Health financing, Equity.

Recebido em: 08.03.2015. Aprovado em: 22.02.2016. 


\section{INTRODUÇÃO}

No Brasil, a saúde é direito de cidadania fundado nos princípios da universalidade, integralidade e equidade (BRASIL, 2006). A equidade é evocada especialmente para reduzir as disparidades em saúde entre grupos sociais e regiões mediante as políticas públicas (RIZZOTTO; BORTOLOTO, 2011). No Sistema Único de Saúde (SUS), a equidade é categoria central do contexto normativo, mas as persistentes desigualdades socioeconômicas ainda existentes no Brasil representam uma barreira à concretização do direito à saúde (COMISSÃO NACIONAL SOBRE DETERMINANTES SOCIAIS DA SAÚDE, 2008; MIQUILIN et al., 2013; GOES; DO NASCIMENTO, 2013; WORLD HEALTH ORGANIZATION, [20--?]).

Nesse contexto, os avanços na ampliação do acesso da população à Atenção Primária à Saúde (APS) (LIMA-COSTA; LOYOLA FILHO; MATOS, 2007) implicam o reconhecimento de que as políticas de saúde têm produzido equidade horizontal, segundo a qual populações com necessidades semelhantes tem obtido acesso análogo aos serviços e ações de saúde (MACINKO; LIMACOSTA, 2012). No enfrentamento das disparidades em saúde, que também requer dos governos a atuação sobre os determinantes sociais da saúde (DSS) (PEARCY; KEPPEL, 2002; MARMOT, 2005), é oportuno discutir o potencial do impacto que os sistemas de saúde podem produzir, especialmente em contextos de persistente desigualdade social (SEN, 2002).

A iniquidade em saúde decorre de características sociodemográficas que determinam 0 acesso diferenciado aos serviços de saúde ou quando diferenças nas condições de saúde decorrem das restrições de escolhas ou de acesso, caracterizando desigualdades injustas (CENTRE FOR HEALTH EQUITY TRAINING RESEARCH AND EVALUATION, 2000). Nesse contexto, a APS é o modelo mais efetivo para a universalização do acesso à saúde com equidade, garantindo também a integralidade, continuidade e qualidade da atenção (STARFIELD, 2004; 2004a).

No Brasil, o Ministério da Saúde (MS) buscou a universalização da APS e instituiu, em março de 2006, a Política Nacional de Atenção Básica (PNAB), mediante a Portaria do Ministro da Saúde $n^{0}$ 648/2006, onde a APS foi denominada Atenção Básica (AB) e a equidade foi incorporada como fundamento dos cuidados primários e promoção da saúde, juntamente com a integralidade, a longitudinalidade e a participação social, dentre outros. Na PNAB, a $A B$ é análoga à definição internacional de APS, representando a operacionalização de um conjunto de ações, no âmbito individual e coletivo, para a promoção e proteção da saúde, prevenção de agravos, diagnóstico 
precoce de doenças, tratamento, reabilitação e manutenção da saúde (BRASIL, 2006a).

A implementação da PNAB tem financiamento tripartite, mas o suporte federal é realizado pelo Fundo Nacional de Saúde (FNS), mediante os repasses dos recursos do Bloco da Atenção Básica (BLATB), de forma regular e automática para os municípios e Distrito Federal. O BLATB é composto pelo Piso da Atenção Básica Fixo (PAB Fixo), de caráter per capita, e o Piso da Atenção Básica Variável (PAB Variável), cujo volume e composição estão condicionados à adesão formal dos municípios e do Distrito Federal aos programas e ações incentivados pelo Ministério da Saúde, no âmbito da PNAB, funcionando como ferramenta de estímulo e coordenação para a implantação das estratégias nacionais de reorganização do modelo de atenção à saúde (BRASIL, 2006a; 2011)

A Estratégia Saúde da Família (ESF) foi consagrada na PNAB como 0 principal modelo de APS/AB no Brasil. A Saúde da Família foi criada em 1994 pelo MS como um programa de incentivos descentralizados dirigido à esfera municipal de governo. Seu objetivo inicial foi favorecer a estruturação/reorganização dos sistemas locais de saúde para a superação do modelo assistencialista vigente centrado na atividade médico-hospitalar (BRASIL, 1998; VIANA; DAL POZ, 1998).
A ESF consolidou-se como modelo de $A B$ e ampliou sua abrangência no país, alcançando em 2011 a 95\% dos municípios brasileiros e $53 \%$ da população (BRASIL, 2014). No Nordeste brasileiro a presença da ESF é superior às outras regiões, sendo que o Piauí apresenta a maior estimativa de cobertura populacional do modelo (99\%) (BRASIL, 2014), sugerindo elevada importância da PNAB para 0 avanço do acesso à saúde naquele Estado, especialmente para os grupos mais afetados pelas desigualdades socioeconômicas.

A implementação da PNAB no Piauí e a evolução do acesso à $A B$ no contexto dos seus municípios são os temas abordados neste estudo, que analisou os anos de 2006 a 2011 com o objetivo de discutir aspectos relacionados com a equidade em saúde no Piauí, tendo como referência empírica a evolução de indicadores relacionados com a alocação dos recursos federais para a saúde e o acesso às ações da PNAB, em especial as consultas médicas para a população geral, infantil e de gestantes.

Espera-se contribuir com a identificação de elementos que subsidiem a discussão nacional sobre 0 papel e a importância da $A B$ no contexto brasileiro, bem como para a tomada de decisão relacionada com a PNAB em diferentes níveis, especialmente para alcançar a efetivação da universalização do acesso à saúde com equidade.

R. Pol. Públ., São Luís, v. 20, n 1, p. 19-32, jan.jun. 2016. 


\section{MÉTODOS}

Este estudo adotou enfoques complementares para subsidiar a discussão sobre a PNAB e a equidade em saúde no Piauí. Primeiro foram comparadas as estimativas oficiais das coberturas populacionais dos principais programas relacionados com a PNAB, a ESF e o Programa de Agentes Comunitários de Saúde (PACS), e suas variações percentuais, no período de 2006 a 2011, para o Piauí, Nordeste e Brasil.

Em seguida, foram calculados indicadores municipais de alocação dos recursos federais da saúde e acesso às ações da PNAB para os anos de 2006 e 2011. Os resultados foram agregados em quintis de renda média domiciliar per capita municipal, segundo o Censo Populacional de 2010 (INSTITUTO BRASILEIRO DE GEOGRAFIA E ESTATÍSTICA, 2011), e foram calculadas as médias e desvios-padrão para cada estrato, acrescentando-se as médias de população residente e de renda familiar per capita em cada estrato de municípios, para fins de comparação. Foi verificada a significância estatística para cada indicador da série, mediante a aplicação do Teste $\mathrm{T}$ de Student bicaudal, pressupondo-se variâncias equivalentes para os anos de 2006 e 2011. Os resultados foram dispostos em uma tabela comparativa para os anos considerados.
Os indicadores selecionados para esta análise foram: i) cobertura populacional estimada da ESF - Sistema de Informações da Atenção Básica (SIAB); ii) valor per capita anual transferido pelo Fundo Nacional de Saúde - Fundo Nacional de Saúde; iii) média de consultas médicas na Atenção Básica por habitante/ano - Sistema de Informações Ambulatoriais do SUS (SIASUS); iv) média de consultas na Atenção Básica na população de 0-4 anos, por habitante na faixa etária/ano SIAB (BRASIL, 2014); e, v) percentual de cobertura de assistência pré-natal com 7 ou mais consultas - Sistema de Informações sobre Nascidos Vivos (SINASC).

Analisou-se a composição das transferências financeiras federais do BLATB no Estado do Piauí, iniciando pela comparação dos valores anuais per capita médios para este estado e para o Nordeste e Brasil. Na etapa seguinte, se desagregaram os componentes do BLATB dos municípios do Piauí, para verificar e comparar sua composição, valores monetariamente atualizados e suas proporções, nos anos de 2006 e 2011. Os resultados foram dispostos em uma tabela.

Por fim, testou-se a hipótese de que as transferências federais, representadas pelos valores per capita do BLATB, têm caráter equitativo, considerando parâmetros socioeconômicos médios municipais. Para isso, utilizou-se modelo de regressão linear múltipla (nível de confiança de 95\%) dos valores per capita anuais do BLATB dos municípios 
piauienses, sobre os fatores socioeconômicos municipais selecionados. Nesta etapa incluíram-se apenas os dados dos anos de 2007 a 2010, em face da indisponibilidade dos anos de 2006 e 2011 no momento da coleta. 0 modelo de regressão incluiu como variáveis explicativas os seguintes aspectos socioeconômicos, definidos e obtidos a partir do Censo Demográfico de 2010 (INSTITUTO BRASILEIRO DE GEOGRAFIA E ESTATÍSTICA, 2011): i) renda média domiciliar per capita mensal; ii) taxa de analfabetismo; e, iii) proporção de pessoas com baixa renda.

Foi realizada a plotagem dos resíduos da regressão, a fim de avaliar a linearidade das relações entre variável independente e explicativas, bem como se os erros foram estatisticamente independentes e distribuídos normalmente. Os resultados foram dispostos em tabelas sintéticas com os resumos dos resultados da regressão.

A coleta de dados foi realizada no período de outubro de 2013 a abril de 2014, junto às bases do Departamento de Informática do Ministério da Saúde (DATASUS) e IBGE e a análise dos dados foi realizada com apoio nos softwares Microsoft Excel ${ }^{\circledR}(2010)$, Stata MP 10 for Windows ${ }^{\circledR}(2009)$ e Datasus-TabWin $3.6^{\odot}$ (2011).

Esta pesquisa utilizou bases de dados secundários de domínio público, em face do que não se considerou a necessidade de submissão a comitê de ética, no entanto, buscou-se seguir os princípios éticos e de confidencialidade de informações recomendados pela Resolução n. 466/2012 do Conselho Nacional de Saúde.

\section{RESULTADOS}

A Tabela 1 apresenta as estimativas de coberturas médias dos programas ESF e PACS para o Piauí, Nordeste e Brasil, e sua variação no período de 2006 a 2011, mostrando que no Piauí estas foram muito superiores às encontradas na região e país. Observa-se também que a expansão dos programas no Piauí foi proporcionalmente

Tabela 1 - Cobertura populacional estimada (\%) do PACS e ESF, Piauí, Nordeste e Brasil, 2006 a 2011

\begin{tabular}{|c|c|c|c|c|c|c|c|c|c|c|c|c|c|c|}
\hline \multirow[b]{2}{*}{ Cenário } & \multicolumn{2}{|c|}{2006} & \multicolumn{2}{|c|}{2007} & \multicolumn{2}{|c|}{2008} & \multicolumn{2}{|c|}{2009} & \multicolumn{2}{|c|}{2010} & \multicolumn{2}{|c|}{2011} & \multicolumn{2}{|c|}{ Variação (\%) } \\
\hline & $\begin{array}{c}\text { PAC } \\
S\end{array}$ & ESF & PACS & ESF & PACS & ESF & PACS & ESF & PACS & ESF & PACS & ESF & PACS & ESF \\
\hline Piauí & 98,2 & 95,2 & 98,0 & 94,8 & 99,1 & 96,0 & 99,3 & & 99,5 & & 99,7 & & 1,5 & 1,6 \\
\hline Nordeste & 84,0 & 65,8 & 81,2 & 66,7 & 82,9 & 69,7 & 83,2 & 70,0 & 85,1 & 72,0 & 86,7 & 72,5 & 3,2 & 10,2 \\
\hline Brasil & 58,7 & 45,3 & 57,2 & 46,0 & 59,1 & 48,5 & 60,0 & 49,8 & 61,7 & 51,8 & 63,4 & 53,0 & 8,0 & 17,0 \\
\hline
\end{tabular}

Fonte: BRASIL. Ministério da Saúde. Secretaria de Atenção à Saúde. Departamento de Atenção Básica. Histórico de cobertura da saúde da família. Brasília, DF, 2014. Disponível em:<http://dtr2004.saude.gov.br/dab/historico_cobertura_sf.php>. Acesso em: 7 jan. 2014. 
Tabela 2 - Média, desvio padrão (DP), variação percentual e significância (teste $T$ de Student) de variáveis selecionadas, segundo estratificação de municípios por quintis de renda familiar per capita média, Piauí, 2006 e 2011

\begin{tabular}{|c|c|c|c|c|c|c|}
\hline Estratos municipais & & & & & Var & ção \\
\hline Quintil renda familiar per capita 1 (até $R \$ 193,03$ ) & Média & DP & Média & DP & $\%$ & $p$ \\
\hline População residente (n) & 5.830 & 3.233 & 5.871 & 3.137 & $0,7 \%$ & 0,952 \\
\hline Renda familiar per capita mensal $(\mathrm{R} \$)^{*}$ & 172,44 & 15,68 & - & - & - & - \\
\hline Cobertura estimada da ESF (\%) & 1,02 & 0,15 & 1,03 & 0,16 & $1,5 \%$ & 0,631 \\
\hline Transferências SUS federais per capita $(\mathrm{R} \$)^{* *}$ & 106,73 & 14,02 & 137,48 & 34,41 & $28,8 \%$ & $<0,001$ \\
\hline Consultas médicas por hab/ano ( $\mathrm{n}$ ) & 0,92 & 0,53 & 1,15 & 0,49 & $24,7 \%$ & 0,039 \\
\hline Consultas médicas por hab/ano (0-4 anos) (n) & 1,42 & 0,74 & 1,58 & 0,72 & $11,7 \%$ & 0,291 \\
\hline Cobertura pré-natal com 7 ou mais consultas (\%) & 28,16 & 14,03 & 40,52 & 13,26 & $43,9 \%$ & $<0,001$ \\
\hline Quintil renda per capita 2 (de $R \$ 193,04$ a até $R \$ 215,72$ ) & Média & DP & Média & $\mathrm{DP}$ & $\%$ & $p$ \\
\hline População residente (n) & 5.879 & 3.722 & 6.135 & 3.933 & $4,4 \%$ & 0,751 \\
\hline Renda familiar per capita mensal $(\mathrm{R} \$)^{*}$ & 205,04 & 6,77 & - & - & - & \\
\hline Cobertura estimada da ESF (\%) & 1,04 & 0,15 & 0,97 & 0,17 & $-7,2 \%$ & 0,028 \\
\hline Transferências SUS federais per capita $(\mathrm{R} \$)^{* *}$ & 111,60 & 13,73 & 141,31 & 45,15 & $26,6 \%$ & $<0,001$ \\
\hline Consultas médicas por hab/ano (n) & 1,01 & 0,56 & 1,23 & 1,13 & $21,4 \%$ & 0,254 \\
\hline Consultas médicas por hab/ano (0-4 anos) (n) & 1,52 & 0,83 & 1,63 & 0,98 & $7,3 \%$ & 0,566 \\
\hline Cobertura pré-natal com 7 ou mais consultas (\%) & 31,60 & 15,82 & 40,50 & 13,63 & $28,2 \%$ & 0,005 \\
\hline Quintil renda per capita 3 (de $R \$ 215,73$ até $R \$ 244,87$ ) & Média & $\mathrm{DP}$ & Média & $\mathrm{DP}$ & $\%$ & $p$ \\
\hline População residente (n) & 8.680 & 8.992 & 9.216 & 8.832 & $6,2 \%$ & 0,772 \\
\hline Renda familiar per capita mensal $(\mathrm{R} \$)^{*}$ & 228,36 & 8,03 & - & - & - & - \\
\hline Cobertura estimada da ESF (\%) & 0,98 & 0,18 & 0,99 & 0,19 & $1,0 \%$ & 0,801 \\
\hline Transferências SUS federais per capita $(\mathrm{R} \$)^{* *}$ & 118,00 & 26,20 & 151,96 & 53,49 & $28,8 \%$ & $<0,001$ \\
\hline Consultas médicas por hab/ano (n) & 1,16 & 0,70 & 1,13 & 0,87 & $-2,5 \%$ & 0,861 \\
\hline Consultas médicas por hab/ano (0-4 anos) (n) & 1,51 & 0,75 & 1,62 & 0,91 & $7,1 \%$ & 0,543 \\
\hline Cobertura pré-natal com 7 ou mais consultas (\%) & 37,92 & 15,64 & 45,74 & 15,41 & $20,6 \%$ & 0,019 \\
\hline Quintil renda per capita 4 (de $R \$ 244,88$ até $R \$ 280,56$ ) & Média & DP & Média & DP & $\%$ & $p$ \\
\hline População residente $(\mathrm{n})$ & 9.655 & 8.637 & 9.905 & 8.855 & $2,6 \%$ & 0,892 \\
\hline Renda familiar per capita mensal $(\mathrm{R} \$)^{*}$ & 260,97 & 10,47 & - & - & - & - \\
\hline Cobertura estimada da ESF (\%) & 1,01 & 0,13 & 0,96 & 0,07 & $-4,4 \%$ & 0,039 \\
\hline Transferências SUS federais per capita $(\mathrm{R} \$)^{* *}$ & 109,35 & 17,26 & 163,11 & 33,43 & $49,2 \%$ & $<0,001$ \\
\hline Consulte & 1,07 & 0,71 & 1,14 & 0,50 & $6,2 \%$ & 0,607 \\
\hline Consultas médicas por hab/a & 1,32 & 0,69 & 1,45 & 0,59 & $10,3 \%$ & 0,322 \\
\hline Cobertura pré-natal com 7 ou mais $\mathrm{cc}$ & 34,01 & 18,08 & 46,53 & 14,00 & $36,8 \%$ & $<0,001$ \\
\hline Quintil renda per capita 5 (de $R \$ R \$ 280,57$ até $R \$ 739,85$ ) & Média & $\mathrm{DP}$ & Média & $\mathrm{DP}$ & $\%$ & $p$ \\
\hline População r & 37.557 & 119.183 & 38.787 & 122.170 & $3,3 \%$ & 0,962 \\
\hline Renda familiar per capita mensal $(\mathrm{R} \$)^{*}$ & 356,54 & 86,65 & - & - & - & - \\
\hline Cobertura estimada da ESF (\%) & 0,99 & 0,10 & 0,99 & 0,09 & $-0,5 \%$ & 0,815 \\
\hline Transferências SUS federais per capita $(\mathrm{R} \$)^{* *}$ & 131,18 & 40,63 & 189,19 & 90,13 & $44,2 \%$ & $<0,001$ \\
\hline Consultas médicas por hab/ano (n) & 1,17 & 0,73 & 1,25 & 1,59 & $6,8 \%$ & 0,761 \\
\hline Consultas médicas por hab/ano (0-4 anos) (n) & 1,35 & 0,53 & 1,31 & 0,56 & $-2,4 \%$ & 0,778 \\
\hline Cobertura pré-natal com 7 ou mais consultas (\%) & 38,20 & 18,80 & 45,58 & 14,23 & $19,3 \%$ & 0,038 \\
\hline
\end{tabular}

Fontes: Dados populacionais baseados na projeção intercensitária do Censo Populacional 2010: INSTITUTO BRASILEIRO DE GEOGRAFIA E ESTATÍSTICA. Censos demográficos: sinopse do Censo Demográfico de 2010. Rio de Janeiro, 2011. Disponível em:<http://www.ibge.gov.br/estadosat/ temas.php?sigla=pi\&tema=sinopse_censodemog2010>. Acesso em: 30 ago. 2014.

Notas: * Dados sobre renda baseados no ano de 2010, Censo Populacional 2010, IBGE (2014); cobertura estimada da ESF, SIAB/MS, 2014; transferências federais, FNS, 2014; dados assistenciais, SIASUS/MS, 2014; dados sobre 0 pré-natal, SINASC/ MS, 2014.

** Valores de 2006 corrigidos monetariamente para valores de 2011 pelo INPC (IBGE). 
menor em comparação com os outros dois níveis geográficos.

A Tabela 2 mostra a estatística descritiva (média e desvio padrão (DP)) das variáveis selecionadas, considerando-se a estratificação de municípios por quintis de renda familiar per capita média. Também mostra o valor de $p$, comparando as séries dos anos de 2006 e 2011.

A Tabela 3 apresenta a comparação entre os valores per capita médios das transferências federais do BLATB para 0 Piauí, Nordeste e Brasil, bem como sua variação percentual no período de 2006 a 2011

Tabela 3 - Valores per capita* (R\$ 1,00) das transferências federais da atenção básica aos municípios, Piauí, Nordeste e Brasil, 2006 a 2011

\begin{tabular}{lccccccc}
\hline Cenário & $\mathbf{2 0 0 6}$ & $\mathbf{2 0 0 7}$ & $\mathbf{2 0 0 8}$ & $\mathbf{2 0 0 9}$ & $\mathbf{2 0 1 0}$ & $\mathbf{2 0 1 1}$ & Variação (\%) \\
\hline Piauí & 136,90 & 154,42 & 158,94 & 153,05 & 172,88 & 185,40 & 35,0 \\
Nordeste & 58,89 & 66,65 & 69,29 & 68,34 & 77,70 & 81,84 & 39,0 \\
Brasil & 43,75 & 49,14 & 52,41 & 51,01 & 57,17 & 60,20 & 38,0 \\
\hline
\end{tabular}

Fonte: BRASIL. Ministério da Saúde. Sala de Apoio à Gestão Estratégica. [Transferência]. Brasilia, DF, [20--?]. Disponível em:<http://sage.saude.gov.br/>. Acesso em: 30 ago. 2014.

Nota: *Corrigidos monetariamente para valores em 2011, com base no INPC (IBGE).

Tabela 4 - Valores* e proporção (\%) por sub-bloco das transferências do BLATB, Piauí, 2006 e 2011

\begin{tabular}{|c|c|c|c|c|}
\hline \multirow{2}{*}{ Sub-bloco da Atenção Básica } & \multicolumn{2}{|l|}{2006} & \multicolumn{2}{|l|}{2011} \\
\hline & Valor $(\mathrm{R} \$ 1,00)$ & $\%$ & Valor $(\mathrm{R} \$ 1,00)$ & $\%$ \\
\hline Piso da Atenção Básica Fixo (PAB fixo) & $58.681 .581,61$ & 24,27 & $67.072 .252,95$ & 20,02 \\
\hline Agentes Comunitários de Saúde - ACS & $34.412 .012,47$ & 14,24 & $71.686 .048,64$ & 21,40 \\
\hline Compensação de Especificidades Regionais & - & 0,00 & $9.451 .933,18$ & 2,82 \\
\hline Incentivo Adicional ao PACS & $3.065 .007,68$ & 1,27 & $5.559 .264,55$ & 1,66 \\
\hline Incentivo Adicional PSF & $1.204 .750,73$ & 0,50 & $625.872,00$ & 0,19 \\
\hline Incentivo Adicional Saúde Bucal & $743.155,22$ & 0,31 & $334.152,00$ & 0,10 \\
\hline $\begin{array}{l}\text { Incentivo de Implantação aos Núcleos de Apoio à Saúde da } \\
\text { Família - NASF }\end{array}$ & - & - & $294.902,40$ & 0,09 \\
\hline Incentivo Financeiro para a Atenção à Saúde de Adolescente & - & - & $63.266,11$ & 0,02 \\
\hline Núcleos de Apoio à Saúde da Família - NASF & - & - & $13.221 .811,20$ & 3,95 \\
\hline Programa de Melhoria do Acesso e da Qualidade - PMAQ & - & - & $652.073,76$ & 0,19 \\
\hline Programa Saúde na Escola - PSE & - & - & $9.070 .746,98$ & 2,71 \\
\hline Saúde Bucal - SB & $28.466 .432,26$ & 11,78 & $33.168 .882,24$ & 9,90 \\
\hline Saúde da Família - PSF & $114.078 .940,16$ & 47,19 & $120.067 .655,76$ & 35,84 \\
\hline $\begin{array}{l}\text { Programa de Requalificação de UBS - Informatização e } \\
\text { Telessaúde }\end{array}$ & - & - & $1.646 .998,08$ & 0,49 \\
\hline Programa de Requalificação de UBS - Reformas & - & - & $2.084 .312,19$ & 0,62 \\
\hline Programa de Expansão do PSF - PROESF & $461.514,56$ & 0,19 & - & - \\
\hline $\begin{array}{l}\text { Programa Nacional de Reorientação da Formação Profissional em } \\
\text { Saúde }\end{array}$ & $135.365,25$ & 0,06 & - & \\
\hline Incentivo à Descentralização de Unidade de Saúde da Funasa & $492.729,51$ & 0,20 & - & \\
\hline TOTAL & $241.741 .489,45$ & & $335.000 .172,04$ & \\
\hline
\end{tabular}

Fonte: (BRASIL, [20--?]).

Nota: *Corrigidos monetariamente para valores em 2011, com base no INPC (IBGE). 
A Tabela 4 apresenta os resultados da desagregação do BLATB transferido aos municípios piauienses nos anos de 2006 e 2011. Verifica-se que a parte fixa correspondeu a $24,27 \%$, em 2006 , e a $20,02 \%$, em 2011, com uma redução da participação proporcional de $8 \%$, enquanto os recursos variáveis e de investimento/manutenção, corresponderam à parcela restante. A mais importante fonte de recursos para a Atenção Básica nos dois anos analisados foi a ESF, correspondendo a $47,2 \%$, em 2006 , e a $35,8 \%$, em 2011, do montante global do financiamento federal da $A B$ transferido aos municípios piauienses.

A Figura 1 mostra a proporção dos sub-blocos integrantes da parte fixa e variável na composição do BLATB, comparando os anos de 2006 e 2011, onde se observa o crescimento da importância relativa do componente variável do BLATB.
A Tabela 5 mostra o resumo dos resultados do modelo de regressão linear que teve como variável dependente o logaritmo do valor per capita das transferências federais do BLATB para os municípios do Piauí, que testou a hipótese de que estes valores podem ser explicados pela variação das médias municipais das variáveis socioeconômicas incluídas, considerando os anos de 2007 a 2010.

\section{DISCUSSÃO}

Quando da criação da PNAB, em 2006, o Piauí já contava com ampla cobertura do PSF e PACS, programas precursores da política, os quais se encontravam presentes em 99\% dos municípios piauienses. Assim, o Piauí encontrou-se, em comparação com o cenário regional e nacional, em situação bastante avançada quanto à implementação

Figura 1 - Proporção dos componentes fixo e variável das transferências federais* do BLATB aos fundos municipais de saúde, Piauí, 2006 e 2011

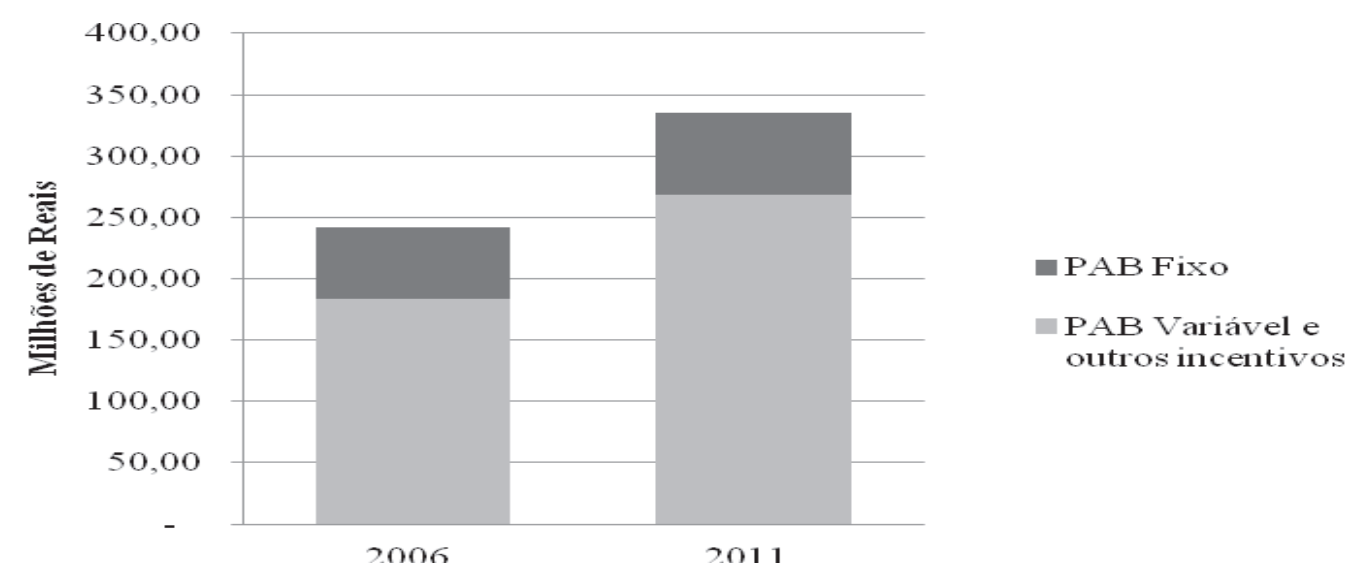

Fonte: (BRASIL, [20--?]).

Nota: *Valores corrigidos monetariamente para 2011, com base no INPC (IBGE). 
Tabela 5 - Resumo dos resultados do modelo de regressão linear múltipla dos valores per capita das transferências federais do BLATB sobre variáveis socioeconômicas, Piauí, 2007 a 2010

\begin{tabular}{lcccccccc}
\hline \multirow{2}{*}{ Variáveis da regressão } & \multicolumn{2}{c}{$\mathbf{2 0 0 7}$} & \multicolumn{2}{c}{$\mathbf{2 0 0 8}$} & \multicolumn{2}{c}{$\mathbf{2 0 0 9}$} & \multicolumn{2}{c}{$\mathbf{2 0 1 0}$} \\
\cline { 2 - 10 } & coef & $\boldsymbol{P}$ & coef & $\boldsymbol{p}$ & coef & $\boldsymbol{P}$ & coef & $\boldsymbol{P}$ \\
\hline Intercepto & 102,84 & 0,003 & 109,54 & 0,007 & 137,68 & $<0,001$ & 161,99 & $<0,001$ \\
\hline Renda média domiciliar & $-0,038$ & 0,329 & $-0,034$ & 0,468 & $-0,056$ & 0,128 & $-0,084$ & 0,072 \\
per capita mensal & 0,180 & 0,461 & 0,579 & $\mathbf{0 , 0 5 0}$ & 0,236 & 0,307 & 0,062 & 0,832 \\
Taxa de analfabetismo & $-0,316$ & 0,345 & $-0,550$ & 0,172 & $-0,556$ & 0,079 & $-0,622$ & 0,121 \\
Proporção de pessoas & $\boldsymbol{F}$ & $\boldsymbol{R}^{\mathbf{2}}$ & $\boldsymbol{F}$ & $\boldsymbol{R}^{\mathbf{2}}$ & $\boldsymbol{F}$ & $\boldsymbol{R}^{\mathbf{2}}$ & $\boldsymbol{F}$ & $\boldsymbol{R}^{\mathbf{2}}$ \\
com baixa renda & 0,492 & 0,011 & 0,097 & 0,028 & 0,164 & 0,023 & 0,235 & 0,019 \\
\hline
\end{tabular}

Fonte: Elaborada pelos autores.

formal do modelo assistencial previsto na PNAB. Isso representa que o PSF fora amplamente aceito como modelo a ser praticado no estado, especialmente se comparado com a região Nordeste e o Brasil, que apresentavam coberturas bem menores, em 2006. Note-se que a expansão das equipes de saúde da ESF requer manifestação expressa dos gestores municipais e suas instâncias de participação comunitária, os conselhos municipais de saúde, para atender aos fluxos burocráticos para o credenciamento e recebimento dos recursos relativos à $P N A B$ (BRASIL, 2006a, 2011). Assim, considerando 0 ano de 2006, já havia pouca margem para uma expansão contínua após a instituição da PNAB, explicando-se assim a pequena variação percentual observada nas coberturas populacionais do estado, entre os anos de 2006 e 2011.

A fim de reduzir os efeitos da heterogeneidade da amostra, julgou-se interessante a estratificação dos municípios piauienses em grupos socioeconômicos, mediante a alocação destes por quintis de renda familiar per capita, para a determinação de indicadores representativos para a PNAB nos diferentes cenários.

Os resultados mostraram que essa abordagem apresenta diferenças importantes no comportamento das variáveis de interesse ao longo do tempo para os diferentes estratos de municípios. A primeira diferença exposta é o limiar de renda no Piauí entre os quintis analisados, estratificados com base nos dados da renda familiar média per capita do Censo Populacional de 2010 (INSTITUTO BRASILEIRO DE GEOGRAFIA E ESTATÍSTICA, 2011). O primeiro quintil foi limitado por uma renda equivalente a $1 / 4$ do limite do quintil mais rico, representando um distanciamento entre os grupos, muito embora os patamares de renda média em todos os quintis se encontrarem abaixo mesmo da renda média nacional (INSTITUTO BRASILEIRO DE GEOGRAFIA E ESTATÍSTICA, 2011).

Outro aspecto importante é a homogeneidade da variável de renda dentro de 
cada estrato analisado. Os desvios padrão observados para as médias municipais podem ser considerados baixos, conduzindo à constatação de que os municípios alocados nos diferentes estratos guardam entre si semelhança quanto a este indicador. Como exemplo, o desvio padrão dentro do grupo de municípios de menor renda familiar per capita foi de 15,68 e as maiores diversidades foram observadas no quintil de maior renda (DP de 86,65 para média de 356,54).

Feita essa digressão sobre as características da renda nos estratos municipais analisados, é preciso se debruçar sobre os achados da comparação dos anos de 2006 e 2011 para as diferentes variáveis estudadas relativas à implementação da PNAB.

Observou-se que duas variáveis apresentaram significância estatística em todos os estratos, as transferências federais do SUS e a cobertura de pré-natal com 7 ou mais consultas. Em nenhum dos estratos a média da população residente variou significativamente, apesar disso foram encontradas diferenças em termos absolutos entre as populações médias dos municípios dos três primeiros quintis e dos dois últimos. A cobertura da ESF, dado estimado com base na população, provavelmente foi influenciada pela atualização populacional do Censo de 2010 (INSTITUTO BRASILEIRO DE GEOGRAFIA E ESTATÍSTICA, 2011) e apresentou variações negativas, exceto no primeiro e terceiro estratos. Já os valores médios das transferências federais do SUS foram ampliados em $49,2 \%$ no terceiro e $44,2 \%$ no quarto estrato, ao passo que nos três primeiros grupos as variações neste indicador ficaram em torno de $28 \%$. Por seu turno, a cobertura de pré-natal com 7 ou mais consultas cresceu $44 \%$ na média dos municípios integrantes do estrato com menor e $19,3 \%$ no grupo com maior renda média familiar per capita.

O comparativo da população residente média nos estratos municipais mostrou grande a diferença entre o quintil de renda mais elevada e os demais. A diferença parece ser influenciada pelos municípios com maior população residente, que também apresentam maior renda média per capita domiciliar. A cobertura estimada da ESF apresentou redução nos quintis 2,4 e 5, se comparados os anos de 2006 e 2011. Importante anotar que apenas no quarto estrato de municípios verificou-se significância estatística da variação desse indicador $(p=0,039)$.

As transferências federais do SUS, por seu turno, cresceram em todos os estratos de municípios no Piauí, no entanto, as maiores variações se deram nos estratos com maior renda per capita familiar. A variação entre os recursos federais do SUS entre 2006 e 2011 indicou que os municípios com maior renda familiar per capita média tiveram maior aumento de recursos, mas é importante ressaltar que esse indicador apresentou significância estatística em todos os estratos 
analisados. A média de consultas médicas por habitante apresentou pouca mudança e nenhuma significância estatística nos estratos e anos analisados, mas o primeiro e segundo grupo por quintis de renda apresentaram maior crescimento, em termos proporcionais. Já nas consultas médicas na população infantil (0 a 4 anos), os quintis inferiores de renda apresentaram vantagens proporcionais aos grupos com renda mais alta. No entanto, essa variação não se mostrou estatisticamente significante nem nos grupos com menor renda per capita, nem naqueles mais favorecidos economicamente.

O indicador relacionado ao prénatal mostrou significância estatística e efetivo aumento de acesso ao serviço de acompanhamento das gestantes, especialmente nos municípios integrantes do grupo com menor renda, onde se verificou 0 maior avanço, 43,9\% de ampliação da cobertura, comparando-se 2006 e 2011. O crescimento nos demais estratos também foi relevante, variando $28,2 \%$, no segundo grupo, $20,6 \%$, no terceiro, $36,8 \%$ no quarto estrato e $19,3 \%$, no grupo dos municípios com maior renda familiar per capita. A cobertura do acompanhamento pré-natal com 7 ou mais consultas cresceu no Piauí no período analisado, alcançando médias aproximadas a $42 \%$ nos diferentes estratos de municípios, refletindo uma ação estritamente relacionada com a PNAB, mas a alta cobertura populacional estimada para a ESF e do PACS pode ensejar que esses resultados sejam considerados como parcialmente satisfatórios, dado que quase a integralidade da população estaria coberta pelas ações da ESF no Estado.

Os resultados da análise das variáveis selecionadas confirmaram que apesar do reduzido potencial de expansão da ESF no estado, alguns aspectos relacionados com a PNAB avançaram de forma importante no período de 2006 a 2011, especialmente quanto às transferências de recursos federais para a saúde e ações de melhoria do acesso das gestantes ao cuidado pré-natal. Os diferentes estratos mostraram resultados heterogêneos, pois as maiores variações positivas de insumos ocorreram nos estratos com maior renda média, ao passo que o acesso cresceu mais nos grupos com menores patamares de renda familiar.

Especificamente quanto à evolução dos repasses federais do BLATB, a evolução dos valores per capita para o Estado do Piauí, no período de 2006 a 2011, apontou 0 crescimento do volume das transferências federais do SUS. O Piauí, efetivamente apresentou valores médios mais elevados, quando comparado à região Nordeste e ao país. Embora o crescimento proporcional destes recursos no estado seja similar aos observado nos níveis regional e nacional, os valores per capita dos repasses, corrigidos pela inflação no período, para o Piauí chegaram a representar até três vezes, em 2006 e 2011, os valores encontrados para o Brasil. 
Se os recursos federais per capita do BLATB do Piauí foram proporcionalmente maiores que aqueles valores encontrados no Nordeste e Brasil no período analisado, a participação do estado no montante nacional dos recursos deste bloco de financiamento federal foi de $4,5 \%$, ao longo do período de 2006 a 2011, representando ao final da série 0 montante de 335 milhões de reais, em valores corrigidos, transferidos pelo governo federal aos municípios piauienses. Neste mesmo período, 2006 a 2011, a parte fixa do BLATB variou em percentuais menores que os recursos da ESF, por exemplo, no montante global do bloco de financiamento federal. Isso mostra a importância que a expansão do PSF teve para que a PNAB encontrasse no Piauí 0 cenário inicial da sua implementação, especialmente quanto ao aumento da disponibilidade de recursos financeiros federais para os municípios desenvolverem as ações de $A B$.

Na questão sobre a dinâmica das transferências federais do BLATB e sua relação com fatores socioeconômicos, os resultados da regressão mostraram que, para os anos de 2007 a 2010, a distribuição dos recursos do BLATB aos municípios piauienses não foi associada com as variáveis socioeconômicas selecionadas, refutando a hipótese de que houve priorização de alocação de recursos federais em cenários com maior carência socioeconômica.
As limitações desta análise residiram no aspecto de que a mesma teve como foco apenas as transferências federais para o nível municipal, sendo esta uma percepção parcial até mesmo do financiamento da $A B$ no Estado, uma vez que os municípios provavelmente ampliaram por meio de recursos próprios a disponibilidade de insumos para as ações da PNAB. Outra questão é que a gestão destes recursos na implementação da PNAB não pode ser considerada homogênea a priori, de modo que as observações direcionadas a comportamentos médios podem não traduzir de forma completa o desenvolvimento real da política nos municípios.

Por fim, a ideia de equidade aplicada a esta análise, pode ser considerada como uma limitação conceitual do estudo, uma vez que considerou que a noção estaria representada in concreto na PNAB mediante a identificação do uso de parâmetros distributivos para orientar a alocação dos recursos federais da $A B$ por condições socioeconômicas, proporcionando maiores aportes nos cenários onde estes recursos potencialmente fossem mais necessários e poderiam proporcionar maiores impactos.

\section{CONCLUSÃO}

Os principais aspectos deste estudo podem ser sintetizados em alguns tópicos especialmente relacionados com os recursos financeiros para a implementação das 
ações da PNAB no âmbito dos sistemas municipais de saúde. Em primeiro lugar, no Piauí, a PNAB representou uma continuidade do PSF e do PACS, cuja expansão se deu de forma ampla nos anos anteriores à instituição da PNAB. Segundo, apesar da melhoria em alguns indicadores de acesso, como o aumento da cobertura do acompanhamento pré-natal com 7 ou mais consultas, a principal mudança observada se deu no âmbito das transferências de recursos federais que cresceram de modo importante, melhorando a disponibilidade de recursos para os municípios. Terceiro ponto, o componente variável do BLATB, em especial os recursos relacionados com a ESF, foi mais importante no contexto da PNAB que 0 componente fixo, apesar do crescimento da disponibilidade de recursos para a saúde. Por fim, não foi possível identificar associação entre as transferências de recursos federais do BLATB e critérios de alocação equitativa, com base nos indicadores socioeconômicos analisados.

\section{REFERÊNCIAS}

BRASIL. Ministério da Saúde. Política Nacional de Atenção Básica. Brasília, DF, 2006a.

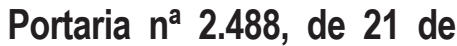
outubro de 2011. Aprova a Política Nacional de Atenção Básica, estabelecendo a revisão de diretrizes e normas para a organização da Atenção Básica, para a Estratégia Saúde da Família (ESF) e o Programa de Agentes Comunitários de Saúde (PACS). Brasília, DF, 2011. em:<http://bvsms.saude.gov.br/bvs/saudelegis/g m/2011/prt2488_21_10_2011.html>. Acesso em: 7 jan. 2013.

Sala de Apoio à Gestão Estratégica. [Transferência]. Brasília, DF, [20-?]. Disponível em:<http://sage.saude.gov.br/>. Acesso em: 30 ago. 2014.

Saúde da Família: uma estratégia para a Reorientação do Modelo Assistencial. Brasília, DF, 1998.

Secretaria de Atenção à Saúde. Departamento de Atenção Básica. Histórico de cobertura da saúde da família. Brasília, DF, $2014 . \quad$ Disponível em:<http://dtr2004.saude.gov.br/dab/historico_co bertura_sf.php>. Acesso em: 7 jan. 2014.

Presidência da República. Senado Federal. Constituição da República Federativa do Brasil de 1988. Brasília, DF, 2006.

CENTRE FOR HEALTH EQUITY TRAINING RESEARCH AND EVALUATION. What is equity? In: An area health service taking action to achieve health for all. Sydney, 2000. Newsletter 1.

COMISSÃO NACIONAL SOBRE DETERMINANTES SOCIAIS DA SAÚDE. Relatório Final. Rio de Janeiro: FIOCRUZ, 2008.

GOES, E. F.; DO NASCIMENTO, E. R. Mulheres negras e brancas e os níveis de acesso aos serviços preventivos de saúde: uma análise sobre as desigualdades. Saúde em Debate, Rio de Janeiro, v. 37, n. 99, p. 571579, out./dez. 2013.

INSTITUTO BRASILEIRO DE GEOGRAFIA E ESTATÍSTICA. Censos demográficos: sinopse do Censo Demográfico de 2010. Rio de Janeiro, 2011. Disponível em:<http://www.ibge.gov.br/estadosat/ temas.php?sigla=pi\&tema=sinopse_censodem og2010>. Acesso em: 30 ago. 2014. 
LIMA-COSTA, M. F.; LOYOLA FILHO, A. I.; MATOS, D. L. Trends in health conditions and use of health services by the Brazilian elderly: a study based on the National Household Sample Survey $(1998,2003)$. Cadernos de Saúde Pública, Rio de Janeiro, v. 23, n. 10, p.24672478, out. 2007.

MACINKO, J.; LIMA-COSTA, M.F. Horizontal equity in health care utilization in Brazil, 19982008. International Journal for Equity in Health, [S. I.], v. 11, n. 33, p. 1-8, jun. 2012.

MARMOT, M. Social determinants of health inequalities. The Lancet, Oxford, v. 365, n. 9464, p. 1099-104, 2005.

MIQUILIN, I. O. C. et al. Desigualdades no acesso e uso dos serviços de saúde entre trabalhadores informais e desempregados: análise da PNAD 2008, Brasil. Cadernos de Saúde Pública, Rio de Janeiro, v. 29, n. 7, p. 1392-1406, jul. 2013.

PEARCY, J. N.; KEPPEL, K. G. A summary measure of health disparity. Public Health Reports, [S. I.], v. 117, n. 3, p. 273-280, may/jun. 2002.

RIZZOTTO, M. L. F.; BORTOLOTO, C. The concept of equity for designing social policies: political and ideological assumptions of the development proposal of CEPAL. Interface Comunicação, Saúde, Educação, Botucatu, v. 15, n. 38, p. 793-803, jul./set. 2011.

SEN, A. ¿Por qué la equidade en salud? Revista Panamericana de Salud Publica, Washington, v. 11, n. 5-6, p. 302-309, maio/jun. 2002.

STARFIELD, B. Atenção primária: equilíbrio entre necessidades de saúde, serviços e tecnologia. Brasília, DF: UNESCO Brasil/Ministério da Saúde, 2004.

Promoting equity in health through research and understanding. Developing
World Bioethics, Oxford, v. 4, n. 1, p. 76-95, 2004a. DOI:10.1111/j.1471-8731.2004.00068.x.

VIANA, A. L. A.; DAL POZ, M. A reforma do setor saúde no Brasil e o Programa da ESF. Physis: Revista de Saúde Coletiva, Rio de Janeiro, v. 8, sup. 2, p. 11-48, 1998.

WORLD HEALTH ORGANIZATION. Global Health Observatory. Statistical Information System. Switzerand, [20--?]. Disponível em:<http://www.who.int/gho/en/>. Acesso em: 13 dez. 2014.

\section{Jorge Otávio Maia Barreto \\ Bacharel em Direito \\ Doutor pelo Programa de Pós-Graduação em Políticas Públicas, Universidade Federal do Piauí (UFPI) \\ Pesquisador em Saúde Pública pela Diretoria Regional de Brasília-DF da Fundação Oswaldo Cruz (FIOCRUZ) E-mail: jorgeomaia@hotmail.com \\ Inez Sampaio Nery \\ Bacharel em Enfermagem \\ Doutora pela Escola de Enfermagem Anna Nery da Universidade Federal do Rio de Janeiro (UFRJ) Professora Associada do Departamento de Enfermagem da Universidade Federal do Piauí (UFPI) e Membro Efetivo dos Programas de Pós-Graduação em Enfermagem e de Políticas Públicas da UFPI \\ E-mail: \\ ineznery.ufpi@gmail.com/pronatuvida@hotmail.com}

\section{Fundação Oswaldo Cruz - FIOCRUZ}

Avenida L3 Norte, Campus Universitário Darcy Ribeiro, Gleba A, SG 10, Brasília/DF CEP 70.904-970

Universidade Federal do Piauí - UFPI

Departamento de Enfermagem, Bloco 12, Campus Universitário Ministro Petrônio Portella, Bairro Ininga, Teresina/PI CEP 64049-550 